\title{
Atomic-Resolution TEM Imaging of Phosphorene Protected by Graphene
}

\author{
Yangjin Lee ${ }^{1,2}$, Jun-Yeong Yoon ${ }^{1,2}$, Hu Young Jeong ${ }^{3}$, and Kwanpyo Kim ${ }^{1,2 *}$ \\ 1. Department of Physics, Yonsei University, Seoul, Republic of Korea. \\ 2. Center for Nanomedicine, Institute of Basic Science (IBS), Seoul, Republic of Korea. \\ 3. UNIST Central Research Facilities (UCRF), Ulsan National Institute of Science and Technology \\ (UNIST), Ulsan, Republic of Korea. \\ * Corresponding author: kpkim@yonsei.ac.kr
}

Phosphorene, a single layer of black phosphorus (BP), is an elemental two-dimensional material with unique properties, such as tunable band-gap and high charge carrier mobility, and exotic in-plane anisotropic physical properties [1]. Atomic resolution imaging of phosphorene, including various atomic-scale defects and edge configurations, is of critically importance but has been elusive due to its degradation under ambient exposure as well as electron-beam-irradiation. To overcome these challenges, we prepared graphene/BP sandwich structures to preserve pristine quality of BP.

Three-layer BP samples were prepared by mechanical exfoliation method. To confirm the layer thickness without surface degradation, optical transmittance of exfoliated BP flakes on a transparent substrate (PDMS) was measured in a $\mathrm{N}_{2}$-filled glove box [2] which is shown in Figure 1(a). Using our previous study, we correlated the local thickness of BP to the optical transmittance [2]. 10\% reduction of transmittance indicates that the local thickness is three-layers. Pre-identified BP flakes and graphene flakes were transferred to $\mathrm{Si}_{3} \mathrm{~N}_{4}$ TEM grid using dry transfer method as shown in Figure 1(c). TEM image of BP/graphene heterostructure sample clearly shows the high quality of BP crystallinity without noticeable structure deformation or residues.

We first study the stability of BP samples under electron-beam-irradiation. Comparison between three different configurations of samples, 1) three-layer BP, 2) three-layer BP/graphene, 3) graphene/threelayer BP/graphene, indicates that graphene lamination results in increase in total exposure electron dose over samples by one order of magnitude. We also observed that graphene protection from only one-side induces the controlled thinning of BP sample. Graphene can reduce e-beam-induced damages to nearby black phosphorus layers, which was demonstrated by previous works on transition metal dichalcogenides[3,4]. The BP layer, which is adjacent to graphene layer, shows the pronounced protection, whereas second/third BP layers can be selectively sputtered out due to reduced protection effect.

Using this controlled thinning process, a pristine single-layer phosphorene was fabricated and atomicresolution imaging was performed (See figure2). Atomic resolution images of phosphorene (from onelayer to three-layer thickness) were compared with TEM imaging simulation, confirming the layernumber-dependent phase contrast.

References:

[1] X. Ling et al., Proc. Natl. Acad. Sci. U. S. A. 112 (2015) 4523.

[2] Y. Lee et al., J Phys. D. J. Phys. D: Appl. Phys. 50 (2017) 084003.

[3] G. Algara-Siller et al., Appl. Phys. Lett. 103 (2013) 203107.

[4] T. Lehnert et al., Appl. Phys. Lett. 110 (2017) 033106. 
[5] The authors acknowledge funding from the Basic Science Research Program through the National Research Foundation of Korea (NRF) funded by the Ministry of Education (NRF-2017R1A5A1014862) and the Institute for Basic Science (IBS-R026-D1).

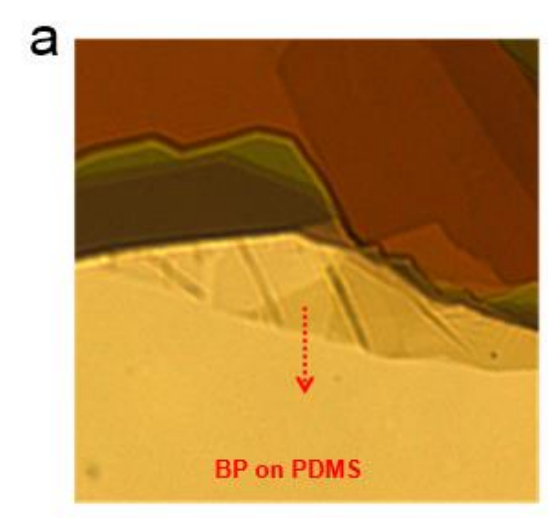

C

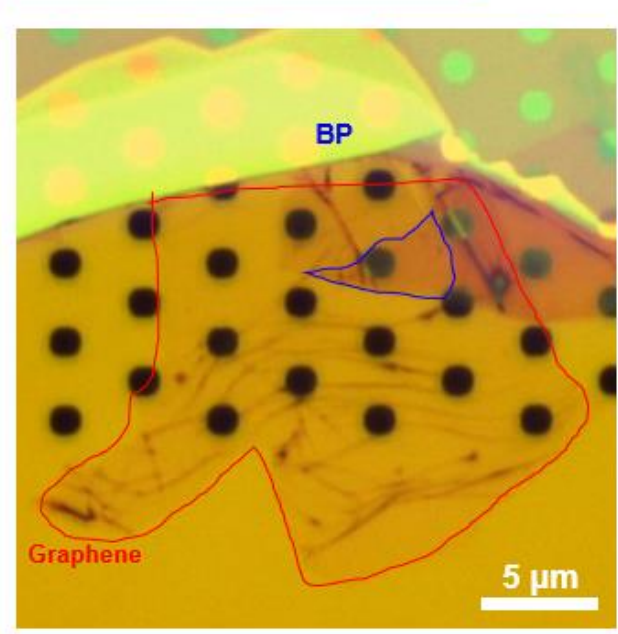

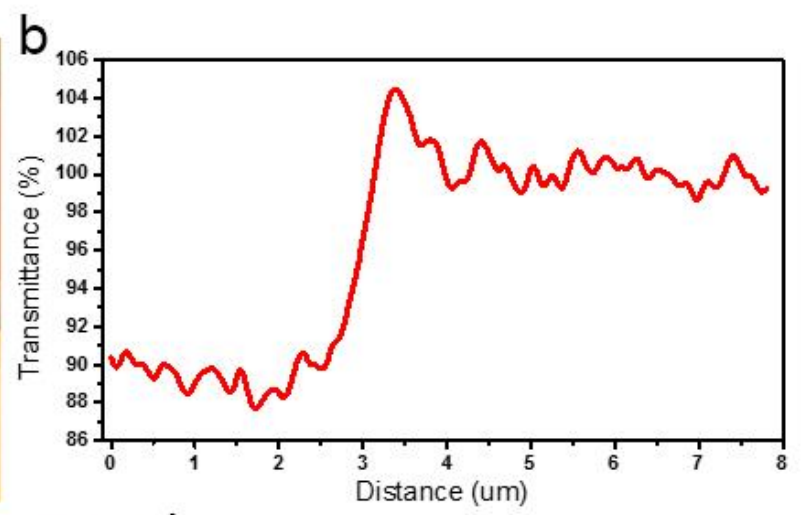

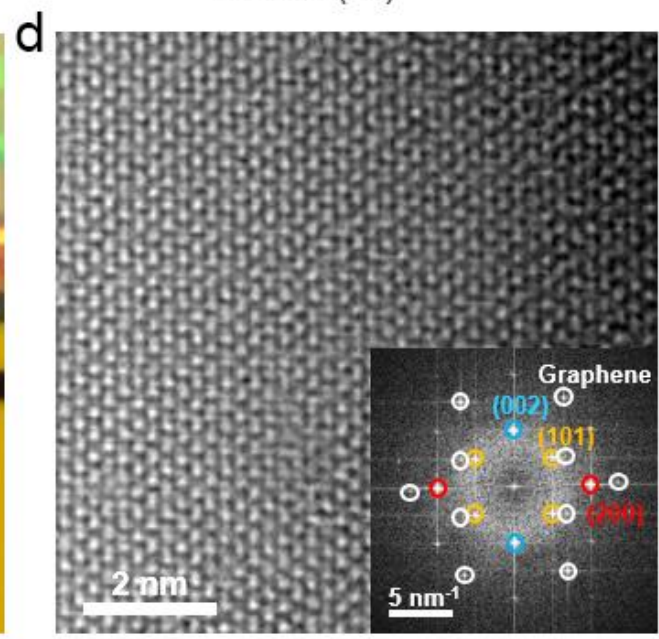

Figure 1. Basic characterization of 3layer BP TEM sample. (a) Optical image of 3layer black phosphorus on PDMS. (b) Optical transmittance along the red arrow in panel a. (c) Optical image transferred 3layer BP/graphene on TEM grid. (d) HR-TEM image of 3layer BP/G. Inset: FFT.
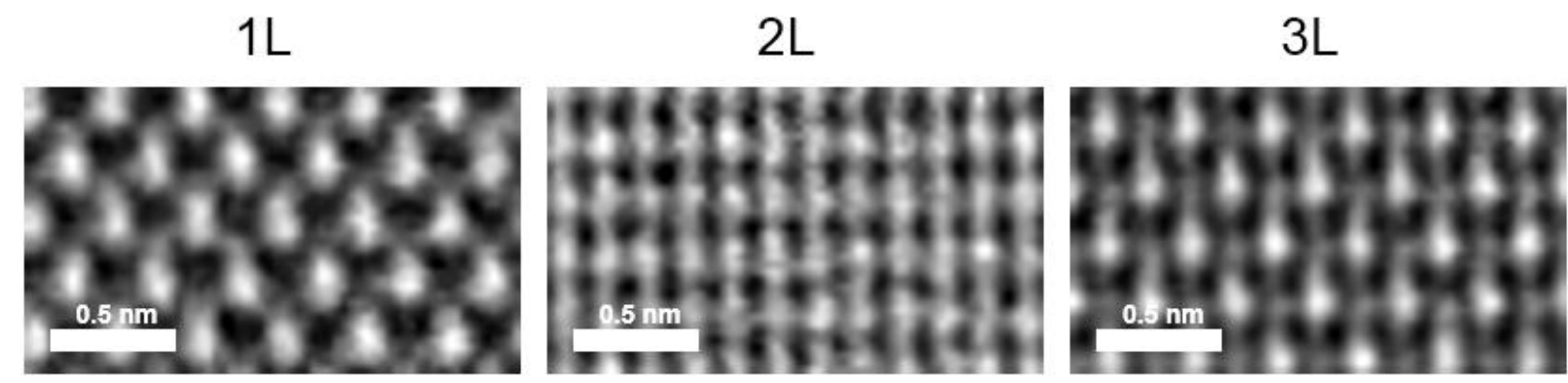

Figure 2. Atomic resolution TEM image of 1,2, and 3layer black phosphorus. 